\title{
Star Formation and Environment in Compact Groups of Galaxies
}

\author{
J. Iglesias-Páramo \\ Instituto de Astrofísica de Canarias, C/ Vía Láctea s.n., 38200 La \\ Laguna, Tenerife, Spain
}

J. M. Vílchez

Instituto de Astrofísica de Andalucía (CSIC), C/ Camino Bajo de Huétor 24, 18080 Granada, Spain

\begin{abstract}
.
$\mathrm{H} \alpha$ luminosities are presented in order to study the Star Formation Rates (SFRs) of a sample of galaxies in compact groups from Hickson's (1982) catalogue. Although the comparison of the SFRs of the disk galaxies in our sample with those of a sample of field galaxies yielded no difference between the average SFRs for disk galaxies in compact groups and in the field, environmental effects seem to influence the $\mathrm{H} \alpha$ luminosities of late and early-type galaxies in compact groups. No relationship was found between the total normalized $\mathrm{H} \alpha$ luminosities of the groups and some dynamical parameters, indicating that the dynamical state of the group does not influence the SFR of the group. The lack of dominant interaction induced starbursts in our sample is compatible with a scenario for compact groups of galaxies in which the dark matter of the group is arranged in a common halo, thereby preventing a fast collapse of the galaxies.
\end{abstract}

\section{Introduction}

Studies devoted to disentangling the nature of compact groups have been carried out by many authors at different wavelength ranges: optical (Moles et al. 1994; Pildis, Bregman \& Schombert 1995), far-infrared (Hickson et al. 1989; Sulentic \& de Mello Rabaca 1993; Venugopal 1995; Allam et al. 1996; Verdes-Montenegro et al. 1998), X-rays (Ebeling, Voges \& Boehringer 1994; Saracco \& Ciliegi 1995; Ponman et al. 1996), and radio (Williams \& Rood 1987; Menon 1995; Huchtmeier 1997).

Understanding the SFRs of galaxies in compact groups could also supply information about their nature. It is known that interactions between disk galaxies can modify their SFRs under some circumstances (Mihos, Richstone \& Bothun 1991). This theoretical result has been observationally confirmed for nearby pairs (Kennicutt et al. 1987; Laurikainen \& Moles 1989) and for samples of peculiar galaxies (e.g. Larson \& Tinsley 1978; Mazzarella, Bothun \& Boroson 1991). Moles et al. (1994), using broadband photometric data, found 

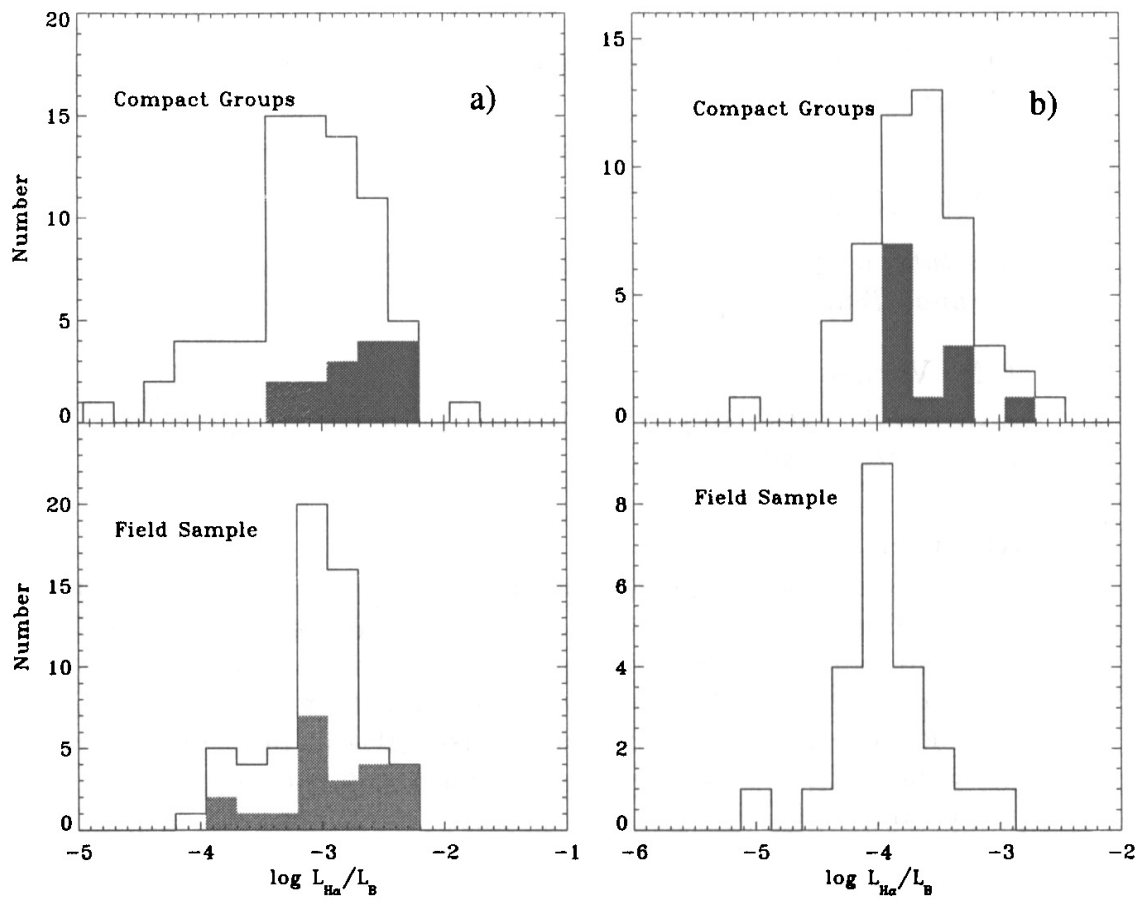

Figure 1. Normalized $\mathrm{H} \alpha$ luminosity histograms for disk galaxies (a) and for early-type galaxies (b). Compact group and field galaxies correspond to top and bottom panels respectively.

a slight enhancement in the SFRs of compact group galaxies compared to field galaxies, suggesting that compact groups of galaxies are not dominated by strong interactions. However, broad band optical colors are only sensitive to the SFRs on time scales of the order of $10^{8} \mathrm{yr}$. A better indicator of the evolution of the SFR on shorter time scales is the luminosity of the hydrogen recombination lines (e.g. Kennicutt 1983). In this work, the $\mathrm{H} \alpha$ luminosities of a sample of galaxies in compact groups are compared to those of a sample of field galaxies in order to study the evolution of the SFR.

\section{The Samples of Galaxies}

Our sample of data was extracted from the Catalogue of Compact Groups of Galaxies (Hickson 1982). It is composed of the two largest compilations of $\mathrm{H} \alpha$ imaging of compact groups of galaxies (Vílchez \& Iglesias-Páramo 1998 , Severgnini et al. 1999). Details concerning the data acquisition and reduction can be found in these papers. A total of 25 complete groups are compiled for this study. Most of the groups have $z<0.02$. 


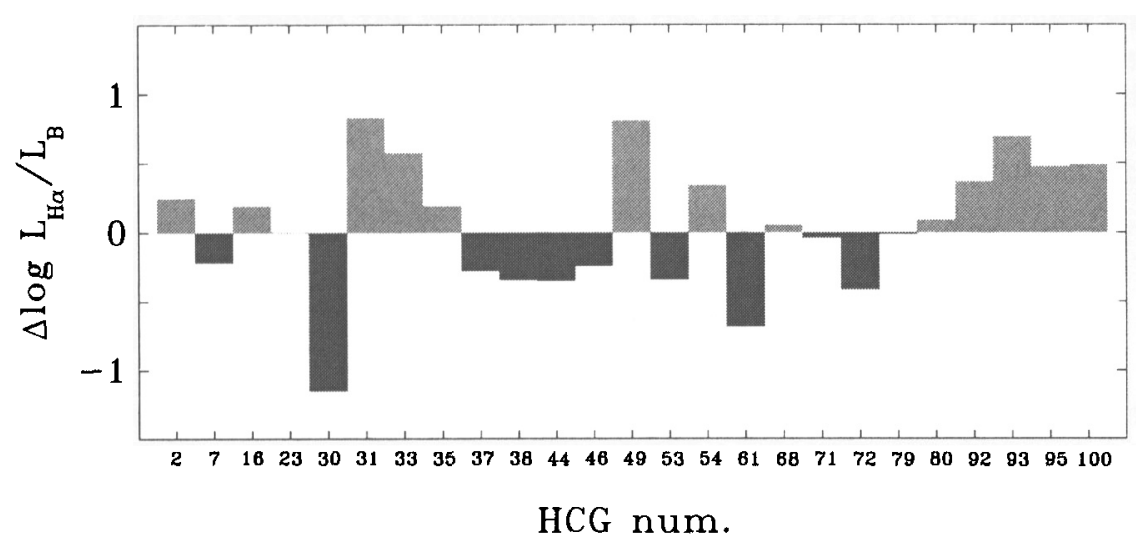

Figure 2. Residual total $\mathrm{H} \alpha$ luminosity normalized to $B$ luminosity for the groups in our sample compared to their expected values.

The comparison sample was taken from three different sources in the literature: spirals from Young et al. (1996), irregulars from Hunter \& Gallagher (1986) and ellipticals and lenticulars from Macchetto et al. (1996). A detailed description of this sample is reported in Iglesias-Páramo \& Vílchez (1999).

\section{Results}

Figure 1a shows the histograms of the $\mathrm{H} \alpha$ luminosities of the disk galaxies normalized to the absolute $B$ luminosities for compact groups (top) and field galaxies (bottom). Grey bins correspond to irregular galaxies in both histograms. Although the mean is indistinguishable for both samples, the upper histogram is significantly broadened with respect to the lower one, with a remarkable tail towards low $\mathrm{H} \alpha$ over $B$ luminosities in the compact group histogram that corresponds to a population of $\mathrm{H} \alpha$-poor spirals in compact groups.

Figure 1b shows the same plot for the early-type galaxies (ellipticals and lenticulars) of both samples. Now, the grey bins in the upper plot correspond to those galaxies which are first-ranked in their respective groups and it can be seen that they are not preferentially found between the most $\mathrm{H} \alpha$-rich galaxies. As can be seen, the average normalized $\mathrm{H} \alpha$ luminosity is significantly brighter for compact group galaxies. We argue that the ionized gas could be the result of accretion processes of free material and/or small satellites during nearby passages with the ellipticals and lenticulars.

Figure 2 shows the difference between the measured and the expected values $^{1}$ of the total $\mathrm{H} \alpha$ over $B$ luminosities for the 25 complete groups in our sample. Positive values are not dominant for our sample of groups. Although most of the groups with $\mathrm{H} \alpha$ over $B$ luminosities larger than expected are

\footnotetext{
${ }^{1}$ Average values supposing that the compact groups were composed by field galaxies with the same morphological types
} 

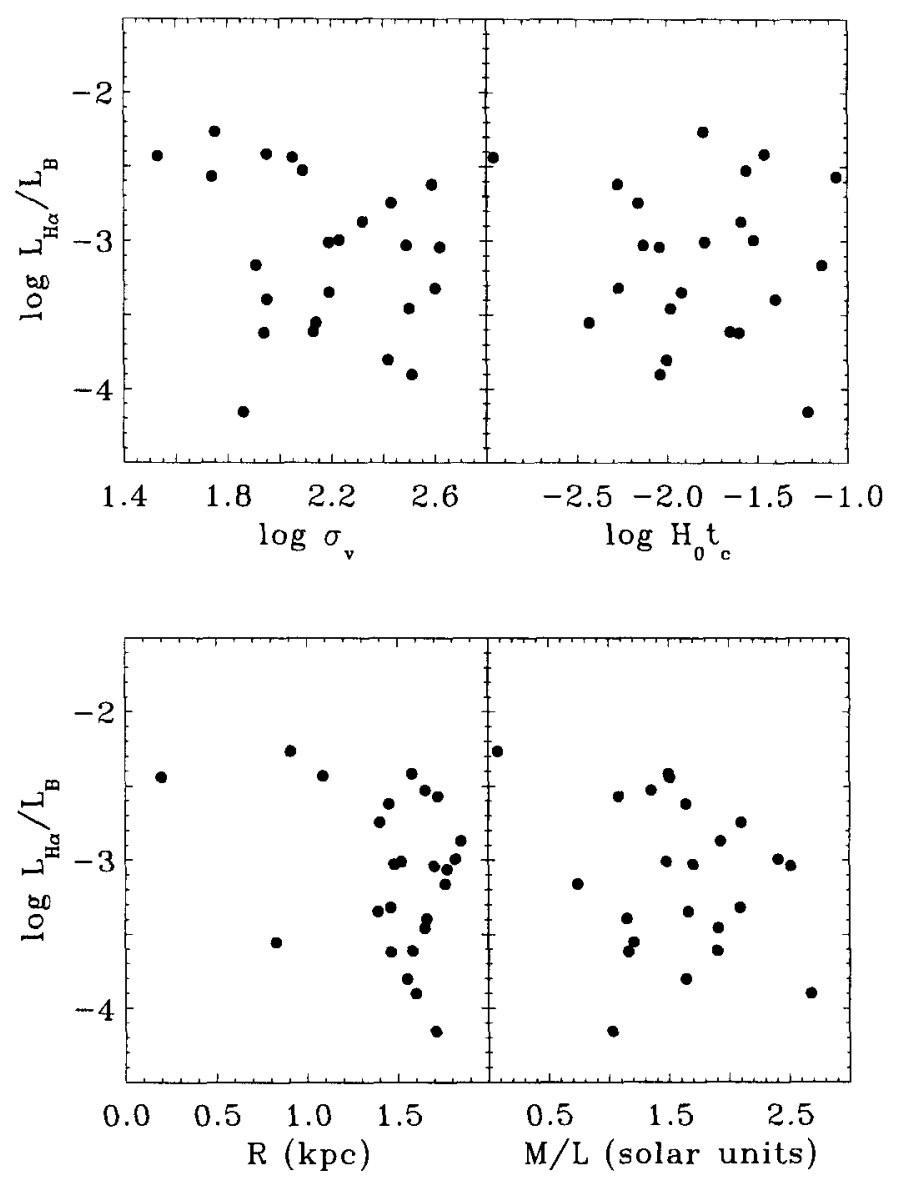

Figure 3. Total $\mathrm{H} \alpha$ luminosity normalized to $B$ luminosity against some dynamical parameters for our sample of groups.

spiral-dominated and/or show strong interactions between disk-galaxies, there are some spiral-rich groups showing low levels of $\mathrm{H} \alpha$ over $B$ luminosities that cannot be explained with the standard picture of ubiquitous interactions within compact groups. Furthermore, it could be expected that those groups with the highest $\mathrm{H}$ I content should show the highest $\mathrm{H} \alpha$ over $B$ luminosities, but such a relationship was not found to hold strongly for the groups in our sample. Perhaps a decoupling between $\mathrm{H}$ I and luminous matter (see Verdes-Montenegro 2000) due to the interactions explain the lack of correlation between both quantities. 
Finally, we studied the relationship of the total SFRs of the groups with their dynamical state. For this purpose we plotted in Figure 3 the normalized $\mathrm{H} \alpha$ luminosities against several dynamical parameters of the groups: velocity dispersion, dimensionless crossing-time, radius and mass to luminosity ratio. No relationship was found in any of the four plots, thus suggesting that the global SFR of the groups is independent of their dynamical state.

\section{Discussion and Conclusions}

In previous sections we have seen that recent SFRs of disk galaxies are not enhanced with respect to the values measured for field galaxies. Although from the environmental point of view, interactions are expected to be one of the most efficient agent affecting star formation in group galaxies, the prediction of the actual effect of interactions on the recent SFR is not straightforward. Published simulations of induced star formation and interactions (Olson \& Kwan 1990; Mihos et al. 1991) predict that only in some cases is the SFR expected to be enhanced during the interaction; the same simulations predict that the SFR could be even depleted for other combinations of parameters describing the interaction. The decoupling of neutral hydrogen and luminous matter detected for some groups could help us to understand the lack of correlation between interactions and enhancement of SFRs in galaxies in compact groups.

The absence of correlation between the total $\mathrm{H} \alpha$ luminosities (as a measure of the SFR) and their dynamical state of the groups could also throw some light on their true nature. Although some simulations show that the lifetimes of compact groups of galaxies should be no longer than a few crossing times (Barnes 1989) and the final fate of compact groups would be a massive elliptical originated by strong interactions and merging of the initial galaxies, observations do not agree with this (Moles et al. 1994, Iglesias-Páramo \& Vílchez 1999). This discrepancy can be explained if we assume that the bulk of the dark matter is mostly arranged in a central halo and shared by all the galaxies in the group instead of being centrally concentrated in the individual galaxies. Recent simulations by Gómez-Flechoso (1997) yielded that if the galaxies are immersed in a dark matter envelope, the lifetime of the group could be as large as $10^{10}$ $\mathrm{yr}$ without substantial changes in the structure of the galaxies except in their outer parts. The assumption that galaxies are composed systems, rather than point particles, coupled with a new mathematical formulation of the dynamical friction, can enlarge the time scales to almost double the classical estimations.

Acknowledgments. Thanks should be given to P. Severgnini and P. Saracco for kindly making available their data. Thanks are also given to Mariángeles Gómez-Flechoso for interesting comments about dynamical friction. The INT and the JKT are operated on the island of La Palma by the RGO in the Spanish Observatorio del Roque de los Muchachos of the Instituto de Astrofísica de Canarias. The $2.2 \mathrm{~m}$ telescope is operated by the MPIA in the Spanish Observatorio de Calar Alto. This research has made use of the NASA/IPAC Extragalactic Database (NED), which is operated by the Jet Propulsion Laboratory, California Institute of Technology, under contract to the National aeronautics and Space Administration (NASA). 


\section{References}

Allam, S., Assendorp, R., Longo, G., Braun, M. \& Richter, G. 1996, A\&AS, 117,39

Barnes, J. E. 1989, Nature, 338, 123

Ebeling, H., Voges, W. \& Boehringer, H. 1994, ApJ, 436, 44

Gómez-Flechoso, M.A. 1997, PhD Thesis, Universidad Autónoma de Madrid

Hickson, P. 1982, ApJ, 255, 382

Hickson, P. , Menon, T. K., Palumbo, G. G. C. \& Persic, M. 1989, ApJ, 341, 679

Huchtmeier, W. K. 1997, A\&A, 325, 473

Hunter, D. A. \& Gallagher, J. S. , III 1986, PASP, 98, 5

Iglesias-Páramo, J. \& Vílchez, J. M. 1999, ApJ, 518, 94

Kennicutt, R. C. , Jr., Roettiger, K. A., Keel, W. C., Van Der Hulst, J. M. \& Hummel, E. 1987, AJ, 93, 1011

Larson, R. B. \& Tinsley, B. M. 1978, ApJ, 219, 46

Laurikainen, E. \& Moles, M. 1989, ApJ, 345, 176

Macchetto, F., Pastoriza, M., Caon, N., Sparks, W. B., Giavalisco, M., Bender, R. \& Capaccioli, M. 1996, A\&AS, 120, 463

Mazzarella, J. M., Bothun, G. D. \& Boroson, T. A. 1991, AJ, 101, 2034

Menon, T. K. 1995, MNRAS, 274, 845

Mihos, J. C., Richstone, D. O. \& Bothun, G. D. 1991, ApJ, 377, 72

Moles, M., Del Olmo, A., Perea, J., Masegosa, J., Marquez, I. \& Costa, V. 1994, A\&A, 285, 404

Olson, K. M. \& Kwan, J. 1990, ApJ, 361, 426

Pildis, R. A., Bregman, J. N. \& Schombert, J. M. 1995, AJ, 110, 1498

Ponman, T. J., Bourner, P. D. J., Ebeling, H. \& Bohringer, H. 1996, MNRAS, 283, 690

Saracco, P. \& Ciliegi, P. 1995, A\&A, 301, 348

Severgnini, P., Garilli, B., Saracco, P. \& Chincarini, G. 1999, A\&AS, 137, 495

Sulentic, J. W. \& De Mello Rabaca, D. F. 1993, ApJ, 410, 520

Venugopal, V. R. 1995, MNRAS, 277, 455

Verdes-Montenegro, L., Yun, M. S., Perea, J., Del Olmo, A. \& Ho, P. T. P. 1998, ApJ, 497, 89

Verdes-Montenegro, L. 2000, these proceedings

Vílchez, J. M. \& Iglesias-Páramo, J. 1998, ApJS, 117, 1

Williams, B. A. \& Rood, H. J. 1987, ApJS, 63, 265

Young, J. S., Allen, L. , Kenney, J. D. P., Lesser, A. \& Rownd, B. 1996, AJ, 112,1903 